\title{
Evaluation of Short-Term Neurological Outcomes in Children with Brain Abscesses
}

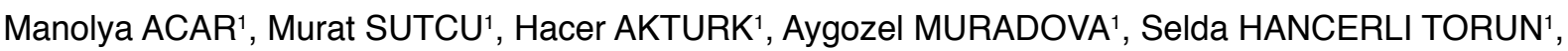 \\ Nuran SALMAN ${ }^{1}$, Mine CALISKAN ${ }^{2}$, Nail IZGI ${ }^{3}$, Ayper SOMER ${ }^{1}$ \\ ${ }^{1}$ Istanbul University, Istanbul Medical Faculty, Department of Pediatric Infectious Diseases, Istanbul, Turkey \\ ${ }^{2}$ Istanbul University, Istanbul Medical Faculty, Department of Pediatric Neurology, Istanbul, Turkey \\ ${ }^{3}$ Istanbul University, Istanbul Medical Faculty, Department of Neurosurgery, Istanbul, Turkey
}

\section{ABSTRACT}

AIM: To evaluate the neurological outcomes of children diagnosed with brain abscesses in the early post-treatment period.

MATERIAL and METHODS: This study was a retrospective analysis of pediatric brain abscess patients between January 2000 and December 2015, during a 16-years period. Patients were divided into two groups according to their outcome at the end of the treatment. The patients with "good outcome" were the ones without any neurological sequelae [Glasgow Outcome Scale (GOS) score 5]. "Unfavorable outcome" was defined as having any kind of neurological deficit (GOS score 1-4).

RESULTS: A total number of 31 patients (22 male, 71\%) with the median age at diagnosis of 84 months (range, 1-202 months) were enrolled in this study. The most common presenting symptom was fever being encountered in $71 \%$ of the patients ( $n=22)$, followed by focal neurological deficit (FND)( $n=17,54.8 \%)$, vomiting $(n=14,45.2 \%)$, headache $(n=13,41.9 \%)$, seizure $(n=13,41.9 \%)$, change in mental status $(n=12,38.7 \%)$ and visual disturbance $(n=2,6.5 \%)$. Twenty-four patients $(77.4 \%)$ had predisposing factors. The most common pathogens were gram-positive cocci $(n=9,29 \%)$. Seventeen patients $(54.8 \%)$ had unfavorable outcome; 2 patients (6.4\%) died. All patients were treated with parenteral antibiotherapy with median duration of 73 days (range, 28-540 days). Surgical procedures were performed in $83.9 \%(n=26)$ of patients [isolated aspiration $(n=19,61.3 \%)$, only resection $(n=5,16.1 \%)$, aspiration and resection $(n=2,6.5 \%)]$.

CONCLUSION: Glasgow coma scale score below 12 and the presence of FND on admission were found to be independent risk factors for unfavourable neurological outcome in children with brain abscesses.

KEYWORDS: Brain abscess, Child, Neurological outcome

\section{INTRODUCTION}

$\mathrm{B}$ rain abscess is a serious life-threatening infection with an estimated incidence of 2-3 cases in every 10,000 hospitalized patients $(6,20)$. Approximately $25 \%$ of all brain abscesses occur in children, especially between $4-7$ years of age $(11,13,18)$. The diagnosis is challenging in pediatric age group, since the presenting signs are often subtle. The classical clinical triad of brain abscess, that is the combination of fever, headache and focal neurological deficit (FND) is observed in only 9-28\% of children (28).
The investigation with advanced radiological modalities and the proper use of antimicrobial agents in addition to surgical intervention led considerable extent of decrease in overall mortality, from up to $40 \%$ to $10 \%$ in recent years. Meanwhile the rate of full recovery has increased from $33 \%$ to $70 \%$ for patients who have been treated effectively (5). Nevertheless, in patients with rapid progression of infection before admission, severe mental status changes such as stupor or coma and rupture of brain abscess into the ventricle, the recovery rate decreases significantly. In these cases, mortality may increase 
up to $80-100 \%$. In addition, neurological sequelae may reach up to $79 \%$ unless the proper treatment is initiated immediately. Seizure is the most common neurological sequela among survivors and particularly observed in patients with frontal brain abscesses $(21,25)$.

For all these reasons, it is crucial for clinicians to be aware of those subtle signs of this uncommon but serious infectious disease, especially in the risk group, in order to provide an outcome without neurological sequela. Although there is a reasonable number of reports regarding brain abscess in adult populations, literature data on pediatric cases are unsatisfactory.

In this study, we analyzed the characteristics of our pediatric patients diagnosed with brain abscesses and aimed to identify the possible predictive factors on their neurological outcome in the early post-treatment period.

\section{- MATERIAL and METHODS}

This study was a retrospective analysis of 31 pediatric patients with the diagnosis of brain abscess between January 2000 and December 2015, during a 16-year period at Istanbul University, Faculty of Medicine, Pediatric Infectious Disease and Clinical Immunology Departments. The characteristics of the patients, predisposing conditions, presenting symptoms and clinical findings, laboratory evaluation, radiological examination including cranial computed tomography (CT) and/ or cranial magnetic resonance imaging (MRI), microbiological analysis, treatment strategies, type and duration of antibiotic therapy, and final outcomes were gathered from patient files.

\section{Definition}

Brain abscess was defined as intraparenchymal collection of purulent material seen on cranial CT and/or MRI, which is surrounded by a contrast rich peripheral ring together with perilesional edema plus either of the following; positive blood cultures, positive culture of abscess material and histopathological confirmation. Subdural and epidural collections were excluded.

\section{Laboratory and Radiological Evaluation}

Laboratory evaluations including absolute leukocyte count, C-reactive protein (CRP) values were recorded. Leucocytosis was defined as white blood cell count $2 \mathrm{SD}$ above age-related normal values (23). CRP levels $>5 \mathrm{mg} / \mathrm{L}$ were accepted as high. Microbiological analysis included the routine aerobic and anaerobic bacteria, mycobacteria and fungi cultures of abscess materials obtained during surgery, blood and cerebrospinal fluid (CSF) obtained by lumbar puncture or external drainage in selective cases or by external drainage. In children with preliminary diagnosis of tuberculosis, gastric lavage fluid culture for Mycobacterium tuberculosis (MTB) was also obtained. In addition, histopathological examination of surgical materials and central nervous system radiological evaluation were performed routinely. Data regarding the localization, size and the number of abscesses, presence of multiple abscesses, perilesional edema and shift were recorded from radiology reports.

\section{Outcome}

Patients were divided into two groups according to their Glasgow Outcome Scale (GOS) score at the end of the treatment. GOS is scored according to following clinical situations; death "1", unresponsive status for weeks or months or until death "2", dependent for daily support by reason of mental or physical disability or both " 3 ", able to work in a sheltered environment and travel by public transportation "4", resumption of normal life; there may be minor neurologic and/ or psychological deficits " 5 ". The patients defined as having "good outcome" were the ones with GOS score 5. GOS score $1,2,3$ and 4 constituted the "unfavorable outcome" group (26).

\section{Statistical Method}

Statistical analysis of data was performed with the Statistical Package for Social Science (SPSS) for Windows version 21.0 (SPSS 21.0, SPSS Inc. USA). Analysis of normality was performed using Shapiro-Wilk and Kolmogorov Smirnov tests. The data were expressed as mean, standard deviation, median, the lowest-the highest value, frequency and percentage. Categorical and numerical variables between groups were compared by using the "Fisher chi-square" and "independent sample t-tests" respectively. Statistical significance limit were accepted as $p<0.05$. The most significant predictors of unfavorable neurological outcome by univariate analysis were chosen. Variables with a $p$ value $<0.05$ in univariate analysis were chosen to perform logistic regression analysis for estimation of independent risk factors.

\section{RESULTS}

\section{Initial Presentation}

A total of 31 patients (22 male, $71 \%$ ) with a median age at diagnosis of 84 months (range, 1-202 months) were enrolled in our study. Average duration of symptoms before presentation was 5 days (range, 1-60 days). General characteristics of our patients were detailed in Table I. The most common presenting symptom was fever, being encountered in $71 \%$ of the patients $(n=22)$, followed by FND $(n=17,54.8 \%)$, vomiting $(n=14$, $45.2 \%)$, headache $(n=13,41.9 \%)$, seizure $(n=13,41.9 \%)$, change in mental status $(n=12,38.7 \%)$ and visual disturbance $(n=2,6.5 \%)$.

On admission, 6 patients (19.4\%) had a positive meningeal irritation sign and 5 patients (16.1\%) presented with cranial nerve palsy. Papilledema and ataxia were encountered in two patients (6.5\%) each. Of all patients, 6 children $(19.4 \%)$ presented with the classical triad of brain abscess (fever, headache and FND). Glasgow coma scale (GCS) score was $<12$ at the time of presentation in 12 patients (38.7\%). Among the patients with good neurological outcome, the incidence of vomiting and the number of children with GCS $\geq 12$ were significantly high ( $p$ values are 0.008 and 0.001 , respectively). On the contrary, the presence of FND on admission was more common among the patients with an unfavourable outcome and this was statistically significant $(p=0.001)$ (Table II). Seven patients $(22.6 \%)$ had leucocytosis whereas 21 children 


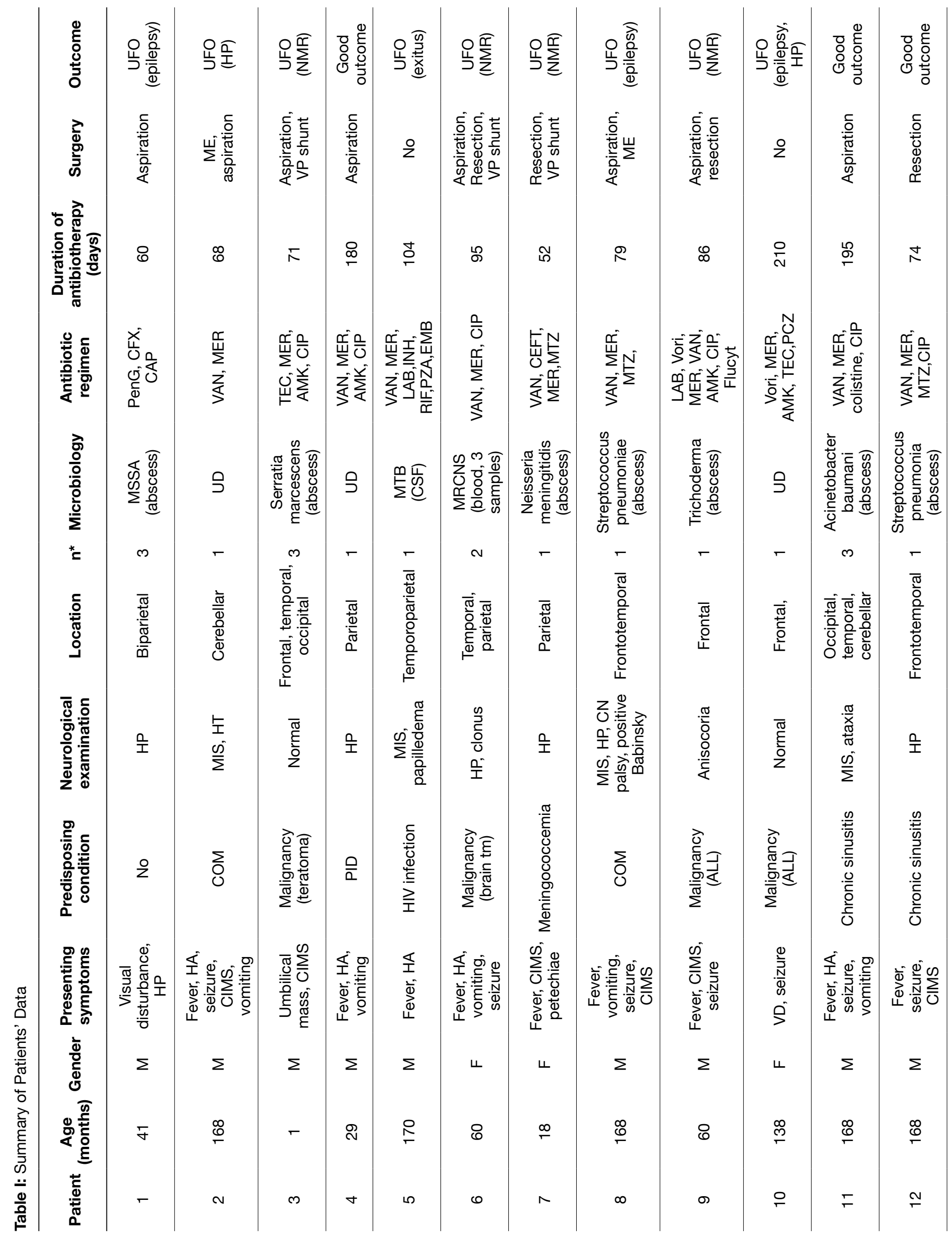




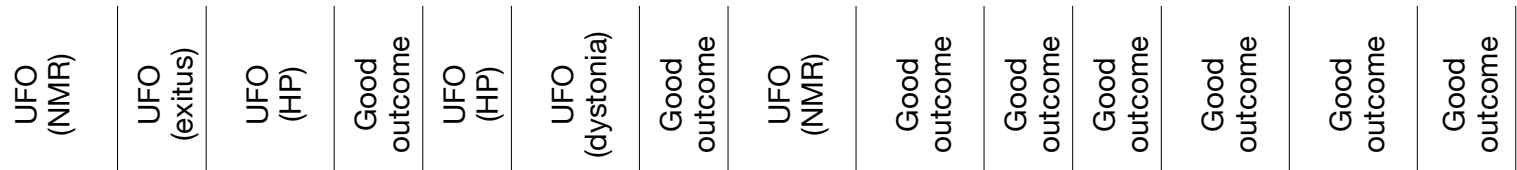

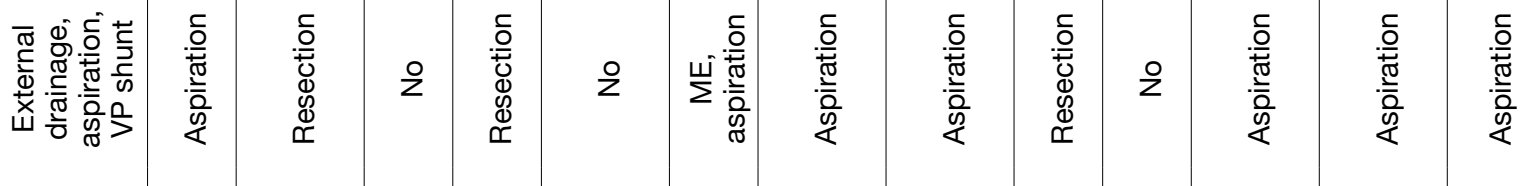

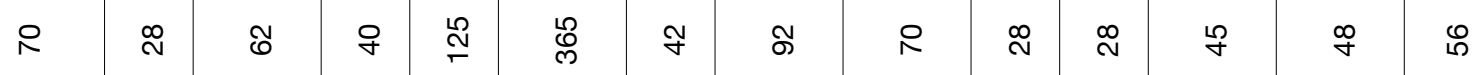

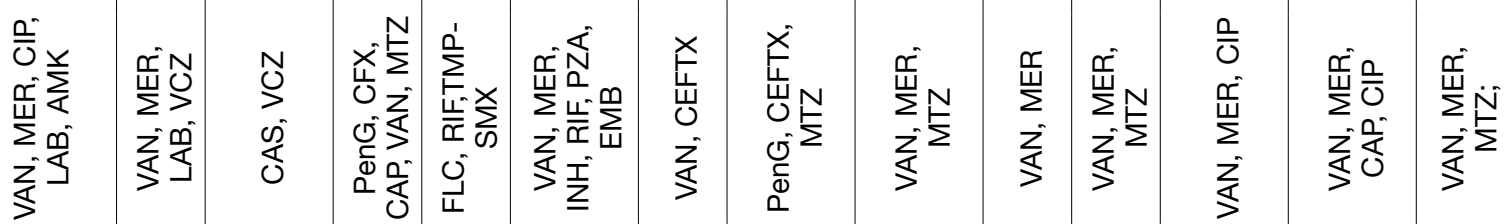

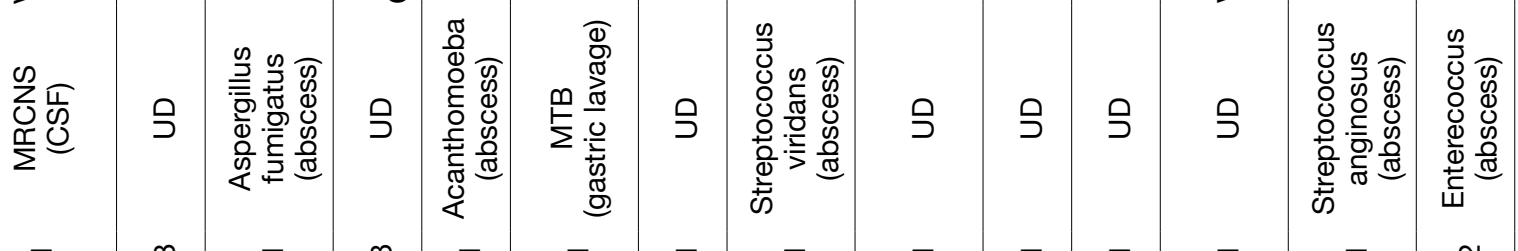

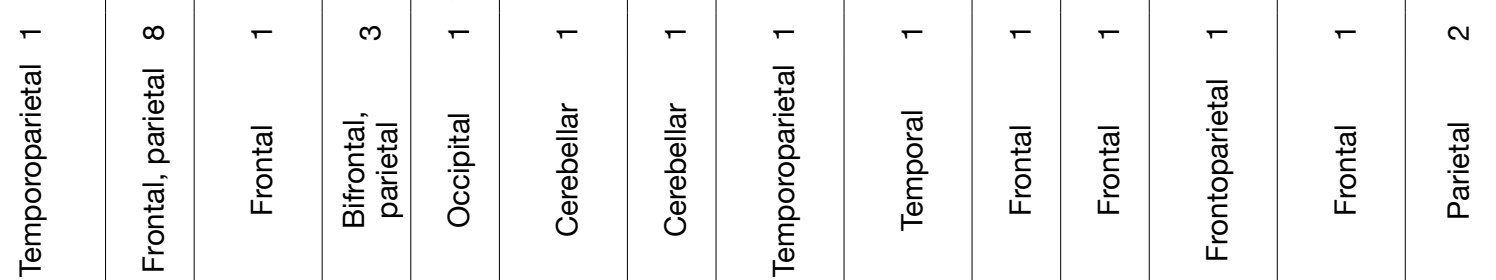

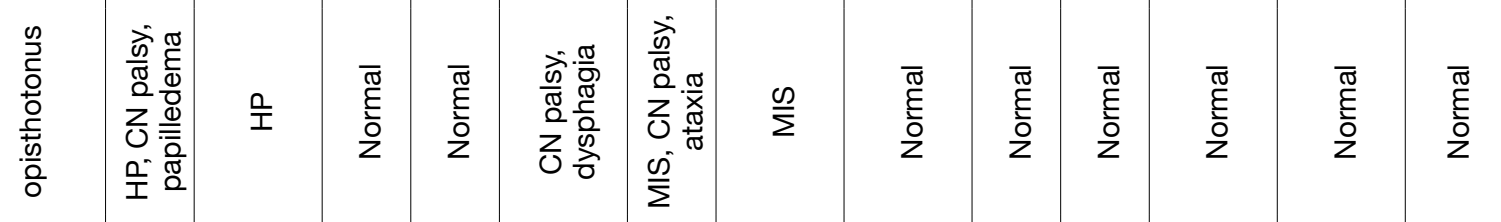

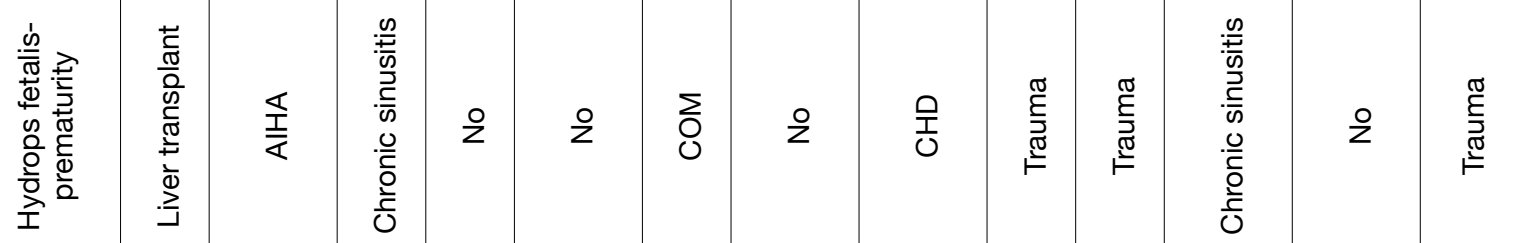

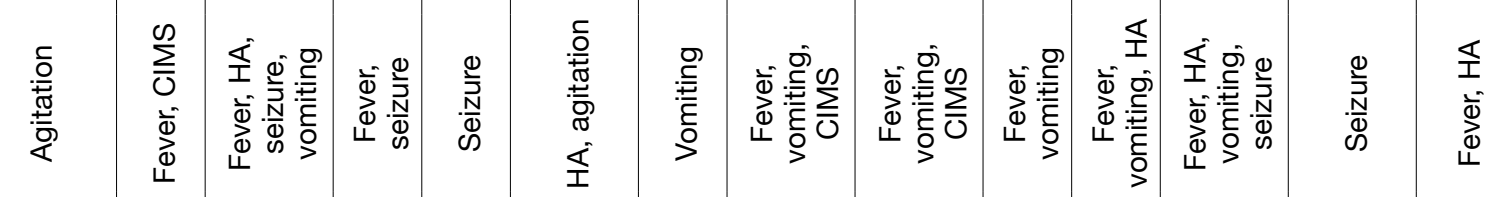

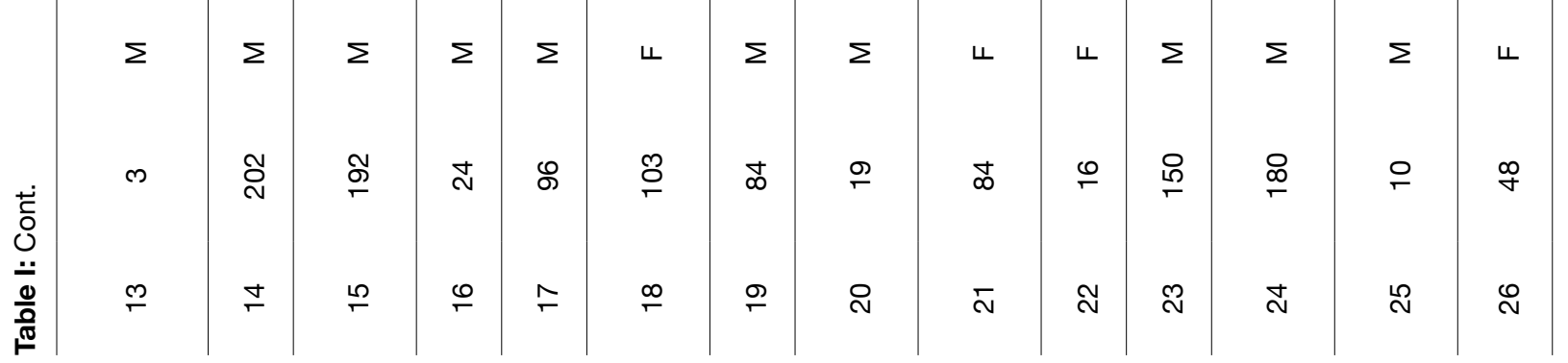


(67.7\%) presented with increased CRP levels. Neither of these conditions was found to be a predictor for neurological outcome.

\section{Predisposing Condition}

Twenty-four patients ( $77.4 \%$ ) had predisposing factors for brain abscess. These were a history of chronic otitis $(n=4,12.9 \%)$, chronic sinusitis $(n=4,12.9 \%)$, penetrating head injury $(n=4$, $12.9 \%)$ and malignancy $(n=4,12.9 \%)$. The malignancies were acute lymphoblastic leukemia (ALL) $(n=2,6.4 \%)$, congenital teratoma $(n=1,3.2 \%)$ and operated brain tumor $(n=1,3.2 \%)$. One patient was diagnosed with primary immune deficiency (PID) whereas 3 patients $(9.6 \%)$ had a secondary immune compromising disorder other than malignancies. Among these, one patient had Human Immunodeficiency Virus (HIV) infection, one patient was under immunosuppressive therapy for liver transplantation, and the last patient was on long-term steroid therapy for autoimmune hemolytic anemia (AlHA). History of premature birth and hydrops fetalis was encountered in a 3-month old boy and 18-month old female presented with brain abscess during meningococcemia. Cyanotic congenital heart disease (CCHD) was rare which was present in two patients $(6.4 \%)$. No underlying predisposing condition could be encountered in 7 children (22.6\%). All of the patients with history of chronic sinusitis as the predisposing factor had a good final neurological outcome in the end $(p=0.018)$.

Hematogenous spread was the most common route for brain abscess ( $n=14,45.2 \%)$ and it was significantly more common among the patients with an unfavourable outcome $(p=0.019)$. Contiguous extension from a primary infectious focus was encountered in 8 patients $(25.8 \%)$ whereas traumatic insemination was the possible cause in 4 children (12.9\%). The dissemination route could not be determined in 5 children (16.1\%).

\section{Properties of the Abscesses}

All patients underwent a head CT and/or cranial MRI. Twelve patients $(38.7 \%)$ had multiple abscesses at the time of presentation. The frontal region $(n=12,38.7 \%)$ and the parietal lobe $(n=7,22.6 \%)$ were the most common localizations for brain abscesses. Less common regions were the temporal lobe, occipital lobe and cerebellum ( $n=4,12.9 \%$, each). The size of the abscess was $\geq 2.5 \mathrm{~cm}$ in diameter in $61.3 \%(\mathrm{n}=19)$ of the patients. No distinction was found between patients with good or unfavourable outcome, in relation to size and location of the abscesses, number of abscesses, presence of intracranial edema or parenchymal shift.

\section{Microbiology}

The microbiological studies included cultures of brain abscess surgical materials, blood, CSF and MTB culture of gastric lavage fluid in a child with concomitant pulmonary tuberculosis and brain abscess. Causative microorganisms could not be determined in 12 patients (38.7\%). The most common pathogens were gram-positive cocci including Methicillin-resistant coagulase-negative Staphylococcus (MRCNS) [( $n=3,9.6 \%)$; one obtained from abscess material, one from CSF, and the last one from 3 different blood cultures], 
Table II: Demographic and Clinical Features of Good and Unfavourable Outcome Groups

\begin{tabular}{lcccc}
\hline Presentation & $\begin{array}{c}\text { Total }(\mathbf{n} \%) \\
\mathbf{n = 3 1}\end{array}$ & $\begin{array}{c}\text { Good outcome } \\
\mathbf{n = 1 4}(\mathbf{4 5 . 2} \%)\end{array}$ & $\begin{array}{c}\text { Unfavourable outcome } \\
\mathbf{n = 1 7}(\mathbf{5 4 . 8 \% )}\end{array}$ & $\mathbf{p}$ \\
\hline Male (n,\%) & $22(71)$ & $10(71.4)$ & $12(70.6)$ & 0.95 \\
\hline Age [months, median (range)] & $84(1-202)$ & $84(10-186)$ & $86(1-202)$ & 0.84 \\
\hline Duration of symptoms [days, median (range)] & $5(1-60)$ & $7.5(2-60)$ & $5(1-50)$ & 0.84 \\
\hline Focal neurological deficit & $17(54.8)$ & $4(28.6)$ & $13(76.5)$ & $\mathbf{0 . 0 0 8}$ \\
\hline Vomiting & $14(45.2)$ & $10(71.4)$ & $4(23.5)$ & $\mathbf{0 . 0 0 8}$ \\
\hline GCS score <12 & $12(38.7)$ & $1(7.1)$ & $11(64.7)$ & $\mathbf{0 . 0 0 1}$ \\
\hline Chronic sinusitis & $4(12.9)$ & $4(28.6)$ & 0 & $\mathbf{0 . 0 1 8}$ \\
\hline Hematogenous spread & $14(45.2)$ & $3(21.4)$ & $11(64.7)$ & $\mathbf{0 . 0 1 9}$ \\
\hline $\begin{array}{l}\text { Duration of antibiotic treatment [days, median } \\
\text { (range)] }\end{array}$ & $73(28-540)$ & $63(28-540)$ & $75(28-510)$ & 0.84 \\
\hline Surgical treatment & $26(83.9)$ & $12(85.7)$ & $14(82.4)$ & 0.59 \\
\hline
\end{tabular}

Methicillin-sensitive Staphylococcus aureus (MSSA) $(n=1$, $3.2 \%$, abscess material), Streptococcus pneumoniae $(n=2$, $6.4 \%$, abscess materials), Streptococcus anginosus $(n=1$, $3.2 \%)$, Streptococcus viridans $(\mathrm{n}=1,3.2 \%)$ and Enterococcus $(n=1,3.2 \%)$ in abscess materials from separate patients. Gram-negative microorganisms such as Serratia marcescens, Neisseria meningitidis, Acinetobacter baumannii and Pseudomonas aeuruginosa were also determined from surgical materials. Mycobacterium tuberculosis was encountered in 3 patients (9.6\%). One of those patients was diagnosed with HIV infection whose CSF culture revealed MTB. No predisposing condition could be found in the other two. Tuberculous abscess was confirmed by histopathological examination in the second patient. For the third patient who had been diagnosed with pulmonary tuberculosis and cerebellar brain abscess, gastric lavage material yielded MTB growth. A 5-year-old boy with a prior diagnosis of ALL presented with a frontal brain abscess that revealed Trichoderma after surgery. Aspergillus fumigatus and Acanthamoeba were the other determined pathogens. The former was encountered in a 16-year old boy with history of AHA and an 8-year old male with no known predisposing condition presented with occipital lobe abscess that yielded Acanthamoeba on histopathological examination.

\section{Treatment}

All patients were treated with parenteral antibiotherapy with a median duration of 73 days (range, 28-540 days). In general, broad spectrum antibiotics, a $3^{\text {rd }}$ generation cephalosporin (ceftriaxone or cefotaxime) plus vancomycin and metronidazole combination or vancomycin plus meropenem were preferred. The antibiotherapy was adjusted according to antibacterial susceptibility of the detected microorganism. Ciprofloxacin was the treatment of choice when tapering oral therapy. Initial four-drug regimen including isoniazid (INH), rifampicin (RIF), pyrazinamide (PZA) and ethambutol (ETM) for 2 months, continued by INH and RIF up to 540 days, was administered to the patients with tuberculous abscesses. The patients with proven (Aspergillus fumigatus and Trichoderma $s p$ ) and those with suspected but undetermined fungal etiology received antifungal therapy. The combination of penicillin $G$ and chloramphenicol had been used earlier, but was not preferred after the widespread use of broader spectrum antibiotics.

Surgical procedures were performed in $83.9 \%(n=26)$ of the patients. Among these, isolated aspiration was performed in 19 patients $(61.3 \%)$ [free-hand aspiration $(n=15,48.4 \%)$ and CT-guided aspiration $(n=4,12.9 \%)]$, total resection in 7 patients $(22.6 \%)$ either as the first choice with craniotomy $(n=5,16.1 \%)$ or in the following operation after aspiration $(n=2,6.5 \%)$. Image-guided or free-hand aspirations were the primarily preferred methods for multiple abscesses and for the abscesses in eloquent brain areas. Concomitant mastoidectomy was performed for the patients with chronic otitis media $(n=4,12.9 \%)$. Five patients $(16.2 \%)$; were only treated with antibiotherapy. Among these, 4 patients $(23.5 \%)$ were in the unfavourable outcome group, in which one of them died. On follow-up, ventriculoperitoneal shunt operations were performed in 4 patients (12.9\%) with a median time of 45 days (range 28-122). In these patients, CSF examination for microbiological and biochemical (total protein and glucose) analysis was executed before surgery. Shunt revisions were applied in two of them secondary to shunt blockage on the $8^{\text {th }}$ and $19^{\text {th }}$ months, respectively.

\section{Outcome}

Fourteen patients $(45.2 \%)$ with no neurological disability at the end of treatment (GOS score 5) compromised the group of patients with "good outcome". A total of 17 patients (54.8\%) had unfavorable outcome; 2 patients $(6.4 \%)$ died and the rest of the patients had moderate to severe disability including neuromotor retardation, epilepsy, hemiparesis and dystonia. One of the patients that died was a 14-year old boy with concomitant stage 3 HIV and tuberculous infection. 
Table III: Multivariate Analysis of Risk Factors for Unfavourable Outcome

\begin{tabular}{ccc}
\hline Variables & p value & Adjusted OR (95\% CI) \\
\hline GCS $<12$ & 0.006 & $11.9(2.4-22.9)$ \\
\hline Initial focal neurologic deficit & 0.026 & $6.3(1.3-12.4)$ \\
\hline
\end{tabular}

Surgical intervention could not be performed since his medical condition was inappropriate. The other patient had a history of liver transplantation and was on massive immunosuppressive therapy. The etiologic agent could not be determined and he was lost although aspiration had been performed. There was no statistical difference between the medical or surgical treatment, or among any of the surgical methods in terms of neurological outcome. GCS score less than 12 and presence of focal neurological deficit on admission were found to be independent risk factors for unfavourable neurological outcome in the early post-treatment period (Table III).

\section{DISCUSSION}

Pediatric brain abscess, although rare, is a serious infectious disorder that may have devastating consequences unless diagnosed and treated effectively. Thenon-specific and obscure manifestations of the disease, especially in small children, leads to diagnostic difficulties and unfavourable neurological outcomes. Several studies have reported headache, fever and altered mental status as the most common presenting symptoms. The incidence of fever exhibits variability among different studies $(6,11,18,20)$. Meyouhas et al. reported that $81 \%$ of the patients had fever at the time of presentation (18). Fever was also the most common presenting symptom in our study $(n=22,71 \%)$. However, since it is very frequent in the pediatric age group, the presence by itself cannot be a reliable sign (10). In addition, secondary to the localized nature of the disease, fever is not the absolute but may be the supporting evidence together with other signs of increased intracranial pressure. Other presenting symptoms in descending order of frequency were FND, vomiting, headache, seizure and change in mental status. The incidences were compatible with the reports of previous studies $(6,18,20)$. Vomiting, as a reflection of increased intracranial pressure was significantly more common among our patients with a favorable outcome. This may be attributable to early detection of the disease because of increased suspicion for underlying cranial pathology. The combination of fever, headache and FND, the classic triad which is an important diagnostic clue for adults, is nonspecific and obscure in children. The incidence is reported as less than $20 \%$ in several studies, similar to our result, being encountered in $19.4 \%$ of all study patients $(18,20,26)$.

Brain abscesses arise secondary to contiguous extension of an infectious focus, mainly chronic otitis media, sinusitis and odontogenic infection, hematogenous dissemination of microorganisms, or trauma. The direct spread of organisms account for $20-60 \%$ of the cases, whereas the source of the infection cannot be determined in $15 \%$ of the cases $(9,12)$. Consistent with the previous data, the number of patients with contiguous infection was $25.8 \%$ in our study. Intracranial complications of otogenic infections have decreased in frequency in developed countries as a reflection of early and proper management of otitis media, although the same is not true for chronic sinusitis $(8,27)$. Carpenter et al. claimed that brain abscesses resulting from a contiguous focus had a better prognosis (7). This data supports our finding that the presence of chronic sinusitis on admission was correlated with a good outcome. This is reasonable since the presence of chronic condition and co-morbidity was high among our study patients. In positive correlation with increased incidence of patients with immune suppression throughout the world, $25.8 \%$ of our patients had an immune compromising disorder such as malignancy (12.9\%), PID (3.2\%), HIV infection (3.2\%) and use of immunosuppressive therapy for other reasons $(6.5 \%)$. The course of infection in this population usually presents with high mortality. In the present study, two patients $(6.5 \%)$, both with underlying immunosuppressive diseases, died during the follow-up period.

Cyanotic congenital heart disease has been determined as a risk factor for children, and declared as the most common disorder in some studies (12). The underlying mechanism is accepted as the presence of ischemic areas in the brain together with increased hemoglobin levels. In the presence of right-left shunting, microorganisms that entered the systemic circulation cannot be cleared within the alveoli $(14,16)$. The rate of $\mathrm{CCHD}$ was $6.5 \%$ in our study cohort, being far below the previous pediatric reports $(6,15,20)$. This may reflect the success of prophylactic antibiotic therapy for infective endocarditis in the risk group as well as the improvements in the operative repair of $\operatorname{CCHD}(5,16)$.

Numerous pathogens can cause brain abscesses depending on the primary site of infection, the age of the patient and the immune status of the host. A meta-analysis of 9699 patients from 123 studies revealed that in $73 \%$ of the pediatric cases the positive culture was monomicrobial, most frequently Streptococcus (36\%) and Staphylococcus spp (18\%) (21). Gram- negative bacteria, encountered in the postsurgical period, following head trauma or otogenic infections are found less often $(16 \%)(4,21)$. Although the surgical material is the most valuable specimen, pus cultures may be sterile in approximately one third of the cases regardless of prior antibiotic use (3). That was also valid for our study and in $38.7 \%$ of the cases no underlying pathogen could be detected. Results of our culture-positive patients were consistent with literature data except for MTB, which was more frequent in our study (9.6\%) (21).

The treatment of brain abscesses requires long-term antimicrobial therapy together with surgical intervention. Medical therapy alone can be considered in single or multiple small 
abscesses ( $<2.5 \mathrm{~cm}$ in diameter) or for patients whom the surgical intervention is inappropriate. In that case, the duration of antimicrobial therapy should last at least $6-8$ weeks $(1,2)$. Multiple abscesses were determined in $38.7 \%$ of the cases and the most common sites were the frontal (38.7\%) and parietal (22.6\%) regions, which was consistent with previous reports (17-20). Nineteen patients (61.3\%) had abscesses $\geq 2.5 \mathrm{~cm}$ in diameter. Intracranial edema and parenchymal shift were encountered in $45.2 \%$ and $22.6 \%$ of the cases respectively and neither of them was found to be correlated with outcome.

In our study, 5 patients (16.2\%), one of them with abscesses smaller than $2.5 \mathrm{~cm}$ in diameter, one patient with multiple small abscesses, two patients with MTB abscesses, and a patient who had been an extremely poor surgical candidate, were only treated with antibiotherapy. Among those, 4 patients were in the unfavorable outcome group, and one of them passed away. Surgical approach mainly consisted of aspiration and/ or resection, together with masteoidectomy for those with underlying chronic otitis. Intraventricular antibiotherapy was administered to $16.1 \%$ of the patients. There was no statistical difference between the medical or surgical treatment, or among any of the surgical methods in terms of short-term neurological outcome.

Previous case series aimed to identify possible predictive risk factors for outcome. Metin et al. reported the positive correlation between initial neurological grade, presence of meningismus, high fever, leucocytosis and mortality (17). Carpenter et al., in their study, mentioned that the patients with underlying cranial neoplasms or medical conditions had worse outcome than contiguous spread (7). Other unfavourable factors were determined as rapid progression of the infection before treatment, presence of multiple abscesses, coma, inadequate treatment, and specific organisms (e.g., Aspergillus species, other fungi, Pseudomonas and Nocardia) in various series $(19,22,24,25)$. In our study, the presence of FND on admission was found as an indicator for unfavourable outcome. Consistent with this finding, $64.7 \%$ of patients with unfavourable outcome had GCS score $<12$ at the time of presentation. When the possible dissemination route was considered, the presence of hematogenic spread was correlated with poorer outcome. No possible correlation was obtained between the size, location, number of the abscesses, or presence of intracranial edema and parenchymal shift among patients in terms of neurological sequelae.

\section{- CONCLUSION}

Brain abscesses are rare but serious infectious disorders and harder to diagnose in children due to the subtle clinical signs. New onset headache, mental status change in addition to fever and signs of increased intracranial pressure should raise the suspicion of brain abscess. Early intervention and appropriate therapy remains the major determinants of patients' outcome. Although medical treatment alone can be preferred in selective cases, surgical intervention is indispensable.

\section{REFERENCES}

1. Alvis-Miranda H, Castellar-Leones SM, Elzain MA, MoscoteSalazar LR: Brain abscess: Current management. J Neurosci Rural Pract 4(1): 67-81, 2013

2. Arlotti M, Grossi P, Pea F, Tomei G, Vullo V, De Rosa FG, Di Perri G, Nicastri E, Lauria FN, Carosi G, Moroni M, Ippolito G: Working group on brain abscess. Consensus document on controversial issues for the treatment of infections of the central nervous system: Bacterial brain abscesses. Int J Infect Dis 14 (4):79-92, 2010

3. Bonfield CM, Sharma J, Dobson S: Pediatric intracranial abscesses. J Infect 71(1):42-46, 2015

4. Brook I: Brain abscess in children: Microbiology and management. J Child Neurol 10(4): 283-288, 1995

5. Brouwer MC, Coutinho JM, van de Beek D: Clinical characteristics and outcome of brain abscess: Systematic review and meta-analysis. Neurology 82(9): 806-813, 2014

6. Canpolat M, Ceylan O, Per H, Koc G, Tumturk A, Kumandas S, Patiroglu T, Doganay S, Gumus H, Unal E, Kose M, Gorkem SB, Kurtsoy A, Ozturk MK: Brain abscess in children: Results of 24 children from a reference center in Central Anatolia, Turkey. J Child Neurol 30(4): 458-467, 2015

7. Carpenter J, Stapleton, Holliman R: Retrospective analysis of 49 cases of brain abscess and review of the literature. Eur $\mathrm{J}$ Clin Microbiol Infect Dis 26(1): 1-11, 2007

8. Chalstrey S, Pfleiderer AG, Moffat DA: Persisting incidence and mortality of sinogenic cerebral abscess: A continuing reflection of late clinical diagnosis. J R Soc Med 84(4):193195, 1991

9. Chun $\mathrm{CH}$, Johnson JD, Hofstetter M, Raff MJ: Brain abscess. A study of 45 consecutive cases. Medicine 65(6):415-431, 1986

10. Finkelstein JA, Christiansen CL, Platt R: Fever in pediatric primary care: Occurrence, management and outcomes. Pediatrics 105(1): 260-266, 2000

11. Ftaziet JL, Ahn ES, Jallo Gl: Management of brain abscesses in children. Neurosurg Focus 24(6): 1-10, 2008

12. Grigoriadis E, Gold W: Pyogenic brain abscess caused by Streptococcus pneumoniae: Case report and review. Clin Infect Dis 25(5): 1108-1112, 1997

13. Helweg-Larsen J, Astradsson A, Richhall H, Erdal J, Laursen A, Brennum J: Pyogenic brain abscess, a 15 year survey. BMC Infect Dis 12(1):332, 2012

14. Infection in Neurosurgery Working Party of the British Society for Antimicrobial Chemotherapy: The rational use of antibiotics in the treatment of brain abscess. Br J Neurosurg 14 (6):525530,2000

15. Lee CG, Kang SH, Kim YJ, Shin HJ, Choi HS, Lee JH, Lee $\mathrm{MH}$ : Brain abscess in Korean children: A 15 year single center study. Korean J Pediatr 53(5):648-652, 2010

16. Mathisen GE, Johnson JP: Brain abscess. Clin Infect Dis 25(4):763-779, 1997

17. Metin F, Akcay SS, Kucukercan M, Berkman MZ, Goktas P: Bacterial brain abscesses: An evaluation of 96 cases. J Infect 52(5): 359-366, 2006 
18. Meyouhas YS, Joseph GB, Guilburd JN, Lorber A, Hadash A, Kassis I: Brain abscess in children-epidemiology, predisposing factors and management in the modern medicine era. Acta Pediatr 99(8): 1163-1167, 2010

19. Nielsen H, Gyldensted C, Harmsen A: Cerebral abscess. Aetiology and pathogenesis, symptoms, diagnosis and treatment. A review of 200 cases from 1935-1976. Acta Neurol Scand 65(6):609-622, 1982

20. Ozsurekci Y, Kara A, Cengiz AB, Celik M, Ozkaya-Parlakay A, Karadag-Oncel E, Ceyhan M: Brain abscess in childhood: $A$ 28 year experience. Turk J Pediatr 54(2): 144-149, 2012

21. Seydoux Ch, Francioli P: Bacterial brain abscesses: Factors influencing mortality and sequelae. Clin Infect Dis 15(3): 394401, 1992

22. Shin JH, Lee HK: Nocardial brain abscess in a renal transplant recipient. Clin Imaging 27(5): 321-324, 2002

23. Stanley F Lo: Reference intervals for laboratory tests and procedures. In: Kiegman RM, Stanton BF, Geme JW, Schor NF (eds). Nelson Textbook of Pediatrics, 20th ed. Elsevier, 2015: 3464-3473
24. Svanteson $\mathrm{B}$, Nordstrom $\mathrm{CH}$, Rausing A: Non-traumatic brain abscess. Epidemiology, clinical symptoms and therapeutic results. Acta Neurochir 94(1-2):57-65, 1998

25. Tattevin P, Bruneel F, Clair B, Lellouche F, de Broucker T, Chevret S, Bédos JP, Wolff M, Régnier B: Bacterial brain abscesses: A retrospective study of 94 patients admitted to an intensive care unit (1980 to 1999). Am J Med 115(2):143146, 2003

26. Tseng JH, Tseng MY: Brain abscess in 142 patients: Factors influencing outcome and mortality. Surg Neurol 65(6):557562, 2006

27. Yen PT, Chan ST, Huang TS: Brain abscess: With special reference to otolaryngologic sources of infection. Otolaryngol Head Neck Surg 113(1):15-22, 1995

28. Yogev R, Bar-Meir M: Management of brain abscesses in children. Pediatr Infect Dis J 23(2):157-159, 2004 